\title{
From 48 to 46 Chromosomes: Origin of Modern Man
}

\author{
A. I. Ibraimov ${ }^{1}$ \\ ${ }^{1}$ Kyrgyz Institute of Balneology and Physiotherapy, Bishkek and Laboratory of Human Genetics, National Center \\ of Cardiology and Internal Medicine, Bishkek, Kyrgyzstan
}

Correspondence: A. I. Ibraimov, Kyrgyz Institute of Balneology and Physiotherapy, Bishkek and Laboratory of Human Genetics, National Center of Cardiology and Internal Medicine, Bishkek, Kyrgyzstan. E-mail: ibraimov_abyt@mail.ru

Received: March 22, 2017

Accepted: March 29, 2017

Online Published: April 6, 2017

doi:10.5539/jmbr.v7n1p80

URL: https://doi.org/10.5539/jmbr.v7n1p80

"Nothing in Biology Makes Sense Except in the Light of Evolution". Th. Dobzhansky

\begin{abstract}
It is old-established that the chromosome number in the chimpanzee, gorilla and orangutan was 48 and not 46 as in man. However, cause and effect of such chromosome rearrangement is unknown. A hypothesis has been proposed that natural selection caused merger of two pairs of autosomes into one chromosome. In the changed climate of the East Africa individuals with less amount of chromosomal Q-heterochromatin regions (Q-HRs) in genome were the most adapted. Two pairs of acrocentrics in the genome the common ancestor, which merged into a single chromosome, apparently, carried on their short arms of Q-HRs with a very high frequency, preventing the birth of individuals with a low number Q-heterochromatin. With the merger of these two pairs of acrocentrics into one, the number of autosomes bearing the Q-HRs reduced from nine to seven pairs, as in the modern human. Such chromosome rearrangement resulted in two important consequences: a) chromosomal Q-HRs distributed into seven Q-polymorphic autosomes, so that it was possible to give birth to the individuals with different, including the low, number of Q-heterochromatin; b) in the population individuals with low number of Q-HRs appeared, able to adapt to new, harsher climatic conditions. With the lapse of time, these individuals formed a new population in the new territory, where individuals with a number of chromosomal Q-HRs like the modern natives of Africa, and with the number of 46 chromosomes in the genome began to dominate. Thus, the cause of the origin of the 46 chromosome karyotype from an ancestral 48 chromosome line was natural selection, and an effect was adaptation, i.e. individuals with different, including the low, number of Q-HRs, got the advantage to open up and to colonize new ecological zones of the East Africa.
\end{abstract}

Keywords: Q-heterochromatin, human adaptation, origin of man

\section{Introduction.}

The modern techniques of differential chromosomal staining (G-, C-, Q- and R-staining) established that the chromosome number in the chimpanzee, gorilla and orangutan was 48 and not 46 as in man. This circumstance, apparently, had some importance in the origin of the modern human. But just which value we still do not know. The range of opinions is quite wide. Briefly, they can be summarized in two extreme opinions: humans appeared after the diploid number of chromosomes in its karyotype became equal to 46, instead of 48 , or became 46 , after the modern human had formed. Since this question is impossible to solve by direct experiment, we can afford to us to speculate freely on this theme without the risk to tread on someone's conceptual mine. But in any case the 48 to 46 was a crucial stage in origin of modern man.

\section{Facts}

As it is known the techniques $\mathrm{G}$ - and R-staining do not reveal existence of hereditary variability at the level of chromosomes in the higher primates. However, these methods are invaluable for exact identification and comparison of fine structure of chromosomes at the microscopic level. Just with their help the similarities and distinctions of chromosomes in karyotypes of the higher primates were found out. These methods allowed us to establish that: a) by a picture of differential staining of chromosomes, a chimpanzee and a gorilla are the closest to a human, than an orangutan; b) the chromosome 2 in a human resulted from merge of two acrocentric chromosomes in the common ancestor of these three higher primates therefore the number of chromosomes instead of 48 became 46 . 
At present several areas uncertainty exist regarding the precise origin of the 46 chromosome karyotype from an ancestral 48 chromosome line. It is assumed that the common ancestor described had 48 chromosomes and therefore ancestral man must have lost two chromosomes. Early proposals (Chiarelli, 1962; Hamerton et al., 1963) pointed out that this might have occurred by centric fusion of two acrocentric chromosomes. Turleau et al. (1972) suggested the idea that the human chromosome 2 has originated de novo from centric fusion of two nonhomologous acrocentrics. However, the techniques G- and R-staining did not helped to receive the answer to a question why there was such chromosomal rearrangement.

In our opinion the most important, in aspect we are interested in, the techniques of chromosomal C- and Q-staining, used for study of heterochromatin areas (HRs) of chromosomes, can give the information. By means of these methods the facts are collected, which, as we hope, can help to construct a hypothesis about an origin of the modern human with the 46 chromosomes. These facts are the follows: 1) Heterochromatin is universally distributed in the chromosomes of all the eukaryotes - plants, animals and man, accounting for $10 \%$ to $60 \%$ of their genome. Heterochromatin regions (HRs) account for about $15 \%-20 \%$ of the human genome; 2) To-date two types of constitutive heterochromatin are recognized: C- and Q-heterochromatin; 3) Despite the fact that chromosomal C- and Q-heterochromatin are defined by a single term, "constitutive heterochromatin", they are undoubtedly significantly different intrachromosomal structures; 4) There are several significant differences between them: Cheterochromatin is found in the chromosomes of all the higher eukaryotes, while Q-heterochromatin - only in man (Homo sapiens), the chimpanzee (Pan troglodytes) and gorilla (Gorilla gorilla); 5) C-heterochromatin regions (CHRs) are known to be invariably present in all the chromosomes of man, varying mainly in size and location (inversion). Q-HRs is detected on certain loci of only seven autosomes in both sexes, as well as on the $\quad \mathrm{Y}$ chromosome of males. Chromosomal Q-HRs in the human genome, unlike those in apes, exhibit considerable quantitative variability; 6) They are localized in pericentric regions, telomere chromosome sections, as well as in regions forming nucleolar organizers (NORs); 7) Broad interspecific and intraspecific variation according to quantitative contents; 8) Ectopic pairing of HRs between homologous and non-homologous chromosomes in interphase nucleus; 9) High break frequency in HR sites or at their border with euchromatin regions 10) They are genetically inert (Hsu \& Arrighi, 1971; Chiarelli \& Lin, 1972; Pearson, 1973; Pearson et al., 1972; Arrighi et al., 1974; Miclos \& John, 1979; Erdtmann, 1982; Prokofyeva-Belgovskaya, 1986; Verma \& Dosik, 1980; Verma, 1988; John, 1988; Bhasin, 2005; Ibraimov, 2015).

However, as it seemed to us, in rearrangement of 48 to 46 chromosomes, apparently, the wide quantitative variability of Q-HRs in the genome of our ancestors played a decisive role. Therefore, we display here some the additional facts, concerning peculiarities of distribution of chromosomal Q-HRs in the genome of populations of the modern human. In particular, it is established:

1) Q-HRs is detected on certain loci of only seven autosomes $(3,4,13,14,15,21$ and 22) in both sexes, as well as on the $\mathrm{Y}$ chromosome of males. On the seven autosomes and the $\mathrm{Y}$ chromosome there are only 13 loci where Q-HRs potentially can be detected;

2) Despite the fact that in the human karyotype there are 13 loci in which Q-HRs can be detected (3cen, 4cen, 13p11, 13p13, 14p11, 14p13, 15p11, 15p13, 21p11, 21p13, 22p11, 22p13, Yq12), i.e., there could theoretically exist individuals with 25 Q-HRs in their genome, but such cases have not as yet been reported;

3) In individuals of a population the number of Q-HRs usually ranges from 0 to 10 . Both complete absence and the maximum number of Q-HRs in the genome have no visible phenotypic manifestations. The presence of individuals in the population with different numbers of Q-HRs in the karyotype (from 0 to 10 ) is due to the fact that Q-HRs are unevenly distributed on seven potentially Q-polymorphic autosomes;

4) Comparative analysis of the native human populations of Eurasia and Africa convinces that the distribution of chromosomal Q-HRs in seven potentially Q-polymorphic autosomes far from accident. In particular, at the level of human populations each of the seven Q-polymorphic autosomes contains comparable "portion" of the overall amount of Q-HRs in population genome irrespective of their race, ethnic, sex and age peculiarities. Indeed, more than a half of Q-HRs are localized in autosomes 3 and 13, and the rest are distributed in autosomes $4,14,15,21$ and $22(29.9 \%, 32.0 \%, 2.6 \%, 6.5 \%, 10.4 \%, 10.8 \%$ and $7.6 \%$, respectively). Hence, if Q-HRs frequencies to be expressed in relative numbers (in percentage from the overall number of chromosomal Q-HRs found in a sample), then it became obvious that interpopulation heterogeneity is formed due to proportional increase or decrease of the absolute number of Q-HRs in all potentially Q-polymorphic loci of seven autosomes simultaneously;

5) The amount of Q-HRs in the genome is best determined by the value of their mean number per individual in a population $(m)$. Consistent interpopulation differences in the quantitative content of Q-HRs in their genome 
were established. These differences proved to be related to features of the ecological environment of the place of permanent residence, and not to racial and ethnic composition of the population;

6) Individuals capable of successfully adapting themselves to the extreme high-altitude climate (e.g. mountaineers) and of the Far North (e.g. oil industry workers of the Jamal peninsula of polar Eastern Siberia) are characterized by extremely low amounts of Q-HRs in their genome;

7) Different age groups have different $m$ values, the greatest number of Q-HRs is characteristic of neonates, while the lowest - of elderly subjects. In the first days, weeks, months and years of life, ceteris paribus, among healthy children the infants often die with the greatest number of Q-HR in genome (Caspersson et al., 1970; Buckton et al., 1976; Lubs et al., 1977; Al-Nassar et al., 1981; Stanyon et al., 1988; Kalz et al., 2005; Ibraimov, 2015; 2016a,b; Ibraimov \& Mirrakhimov, 1982a,b,c,d; 1985; Ibraimov et al., 1982; 1986; 1990; $1991 ; 2013 ; 2014)$.

Distribution of chromosomal Q-HRs on Q-polymorphic autosomes in population of a chimpanzee and a gorilla are absolutely the other. In chimpanzee Q-HRs have been found at the proximal region of the short arm of the acrocentric chromosomes Nos. 14, 15, 17, 22, and 23. 'The frequency of brilliant polymorphisms in the chimpanzee is considerably higher than in man; in man their incidence averages from about 2.9-4.2 (Buckton et al., 1976), while in the chimpanzee it has been estimated to be equal to 8.77 (Lin et al., 1973), and to 8.85 (Seuanez, 1977). ...Q-band polymorphisms are also evident at the telomeres of many chromosomes of the chimpanzee complement. These regions are stained with medium intensity, and variation may then be only in size, but not in their staining intensity. These regions are better demonstrated with C-banding' (Seuanez, 1979).

Concerning the quantity of chromosomal Q-HRs in a gorilla it is known, that: 'In this species, autosomal brilliant Q-band polymorphisms have been found in the centromere region of chromosome 3, the terminal satellites of the acrocentric chromosomes 12,13,14,15, and 16, and at the proximal short arm regions of chromosome 22 and 23. Variations account for change in size and/or fluorescent intensity (Miller et al., 1974). The frequency of brilliant autosomal polymorphisms accounted for 14.8 in specimens of gorilla, which is approximately five times the number observed in man (Seuanez, 1977). ...Brilliant fluorescence is found at the Y chromosome long-arm distal tip in this species. ...Q-band polymorphisms are also evident at the telomeric region of many chromosome arms; these regions which are stained with medium intensity may vary in size between homologue chromosomes and among individuals and can be better demonstrated with C-banding' (Seuanez, 1979).

Despite the presence of the large $\mathrm{Y}$ chromosome in the orangutan intensely fluorescent chromatin is not found in the species or elsewhere in the primate and mammalian kingdom indicating that it must have had a relatively recent origin, that man, chimpanzee, and gorilla form a natural group and that they have had a recent common ancestor (Pearson et al., 1971).

\section{Interpretations}

We believe, that the modern human has appeared because not for the reasons unknown to a science, when at some stage of history in population of our ancestors, the individuals began appear with 46 chromosomes being crossed among themselves, resulted to appearance of the modern human. For example, there are no sufficient bases to believe, that the human could not exist with 48 chromosomes in karyotype. Thus, after merge of two acrocentrics in one submetacentric, loss of euchromatin materials of chromosomes did not happened. Insignificant losses of chromosomal material occurred for the amount of heterochromatin of short arms of two acrocentrics at their merge in one chromosome.

Then, how did the individuals with 46 chromosomes in genome appear in population? We think it highly probable, that change of a climate in the East Africa was the starting mechanism in occurrence of the individuals with 46th chromosomes in population of the modern human ancestors. Middle and Late Miocene ecology was far from being uniform, and such climatic changes as cooling, aridity, seasonal and diurnal temperature fluctuations gradually became dominant environmental factors (Andrews \& van Couvering, 1975). Thus, even before they left Africa our ancestors were probably faced with the problem of adaptation to new, more rigorous natural conditions differing from those of the savannah.

We believe that in appearance of the modern man the possibility of appearance in the population of our ancestors of the individuals with different number of chromosomal Q-HRs, played a decisive role, not the birth of the individuals with the 46th chromosomes. In particular, the two ancestral acrocentric chromosomes, of which chromosome 2 of a human was formed, apparently, had Q-HRs with a very high frequency on the short arms. However, this circumstance did not interfere, though very, in the birth of individuals with different numbers of Q- 
HRs in a population with 48 chromosomes. But, they were eliminated by the natural selection because of their poor adaptability to the hot climate where our common chimps and gorilla ancestors lived.

But, as the number and/or depletion of food increases, populations of the common ancestor began to expand the territory of their habitat to more severe, including cold areas (see above). Perhaps, just at this stage in the life history of our ancestors, the evolutionary changes appeared in the genome, which, ultimately, resulted to the emergence of the modern human. In particular, the individuals with a number of chromosomal Q-HRs in the genome closest to the modern Africans became adapted better in the new climate conditions (see Ibraimov, 1993, 2010, 2011, 2015, 2017).

But wherefrom there are individuals with different, including with a low number of chromosomal Q-HRs in the population of our ancestors? Obviously, some of these individuals, as before, were born from parents with 48 chromosomes. However, due to their low number they could not form a new population, even if they had some adaptive advantage in the new environmental conditions. Here, a fundamentally new solution of the problem was required. Namely, it is the merger of the two pairs of acrocentrics bearing Q-HRs with a very high frequency into one chromosome.

To substantiate such possibility we need to recall the long-established facts in cytogenetics. Thus, before the development of the techniques of differential staining, it was discovered by the routine methods, that the acrocentric chromosomes of human are inclined to association. This means that in the interphase nuclei these chromosomes are joined together with HRs of the short arms. It is known that such associations of acrocentric chromosomes are associated with the formation and functioning of nucleolar organizers in the nucleus. As, the ectopic pairing of HRs between homologous and non-homologous chromosomes in interphase nucleus and high break frequency in HR sites or at their border with euchromatin regions are integral properties of constitutive heterochromatin, why not to assume that such processes could result in the merger of the two pairs of acrocentrics with Q-HRs on the short arms of the common ancestor?

Returning to the question of how did different individuals, including those with a low number of chromosomal QHRs come into the populations; now we can answer that here, apparently, the tendency of acrocentrics to associations played a decisive role. In this case, the merging of two pairs of ancestral acrocentrics allowed: a) to birth the individuals with different, including the low, number of Q-HRs; b) the populations gradually to eliminate two pairs of acrocentrics with a particularly high frequency of Q-HRs. Just the latter circumstance prevented the birth in the population of individuals with different, and especially with a low number of chromosomal Q-HRs, which, as we believe, is better adapted to harsher ecological conditions of the environment (Ibraimov, 1993, 2010, 2011, 2017).

But the aforementioned does not mean that all of the chromosomes, including acrocentrics, only two pairs of acrocentrics with a high frequency of Q-HRs in the genome of the common ancestors could merge to one. It is known that all chromosomes regardless of their form can merge into one. But just acrocentrics often merge into one chromosome because their short arms possess chromosomal HRs and NORs, contributing to the close association in the interphase nucleus.

Now let's see how these facts relate to our interpretation. We postulate that two pairs of acrocentric chromosomes in the genome of the ancestral population, which generated the chromosome 2 of the modern human, were bearers of Q-HRs with a very high frequency. In the genome of the population of the modern human only two pairs of autosomes (3 and 13) are the high frequency, and for more than half of the total amount of chromosomal Q-HRs belongs to their share, and the rest amount of Q-heterochromatin is distributed on the other Q-polymorphic chromosomes $(4,14,15,21$ and 22) with frequency from $3 \%$ to $10 \%$ (see above). Apparently, our ancestors had the number of high Q-polymorphic chromosomes by two pairs more than the modern human. But it wasn't enough. We believe that it is imperative that chromosomal Q-HRs was not distributed equally to all potentially Qpolymorphic autosomes. If the population have uniform distribution of chromosomal Q-HRs, then it will be difficult, if not possible, to birth the individuals with different numbers of Q-heterochromatin in the genome. As the presence of individuals in human population with different numbers of Q-HRs in the karyotype (from 0 to 10) is due to the fact that Q-HRs are unevenly distributed on seven potentially Q-polymorphic autosomes (Ibraimov, 1993, 2011; Ibraimov \& Mirrakhimov, 1985).

As an analogy, we give the example with banknotes on the example of the US dollar. Banknotes of denominations of $1,2,5,10,20,50$ and 100 are in free circulation and use, that is, as many as autosomes, in the human genome that can potentially have a chromosomal Q-HRs. (Of course, we are far from thinking that the Americans, with the choice of the number of banknotes are focused on the human karyotype, as then the very existence of the chromosomes in Nature is unknown). Obviously, they came from common sense, if to print seven banknotes in 
varying proportions (that is, the most is 1 and less of all the 100 dollar bills), then the number of possible combinations at cash transactions be virtually unlimited.

We believe that, after the merger of the two pairs of acrocentrics into one due to the loss of Q-heterochromatin blocks on their short arms, the probability of birth in the population of individuals with different, including the low, number of Q-HRs in the genome has increased significantly. Therefore, it is highly likely that this chromosome rearrangement in the karyotype of the common ancestor ultimately, contributed, to the appearance of the modern man. As it allowed the appearance in the population of individuals with different number of chromosomal Q-HRs in the genome, thereby opened a completely new way of adaptation to the climatic and geographical conditions, different from the East Africa (for more details see Ibraimov, 1993, 2010, 2015, 2017).

It is possible that, sometime in the ancestral population individuals, as the 48 , and the 46 chromosomes could coexist. But with the lapse of time the latter began to prevail over the first, and gradually they were replaced. Most likely, it's happened in the new territory, when the individuals with the 46 chromosome, having some adaptive advantage over individuals with 48 chromosomes in the new climates became dominant in the population numbers.

Then the question is appeared, what is the main at this: a number reduction of the chromosomes from 48 to 46 or something else? We believe that the correct answer will be: both. The modern human could exist with 48 chromosomes in the karyotype, if two pairs of acrocentric chromosomes in the genome of the ancestral populations, wherefrom the later chromosome 2 was formed, did not have at all or had Q-HRs with a very low frequency. However, it was that it was. Just, the existence on their short arms of chromosomal Q-HRs with a very high frequency made it difficult (but not excluded) to give birth in the population to individuals with different, and in particular a very low number of Q-heterochromatin in the genome.

We also state that the modern man without individuals in the population with different, and in particular, the low number of chromosomal Q-HRs would not be able to populate all climatic and geographical zones of Africa and Eurasia (Ibraimov, 1993, 2010, 2011). In this case, the main problem in appearance of the modern human included in deliverance from chromosomes in the gene pool of the ancestors, bearing Q-HRs with a very high frequency, preventing to give birth in the population to individuals with different numbers of Q-heterochromatin. Eventually, this problem was solved by the merger of two high Q-polymorphic (in the meaning of the number of Q-HRs) acrocentrics to one, so that they have lost only parts of their short arms with Q-heterochromatin, but at this their long arms remained intact.

It is hard to say why the ancestors of $P$. troglodytes and G. gorilla were unable to use the same route. However, the assumption which we feel is likely is the following one: initial Q-HRs frequencies on all the variable loci proved to be high enough to produce of individuals with significantly different numbers of chromosomal Q-HRs and, hence, the appearance of individuals with a various numbers of Q-HRs who would be able to survive under unfavourable conditions was quite improbable.

Of course, we are far from thinking that in the genome of our ancestors the other chromosomal rearrangements did not take place, including those, which related to chromosomes without Q-HRs. They, apparently, took place. But in the process of evolution only those rearrangements preserved that were not eliminated by natural selection.

\section{Conclusions}

The nature of chromosomal Q-HRs is obscure. Its renaturation kinetics suggests that it consists of repetitive nucleotide sequences (Weisblum \& de Haseths, 1972). Functionally, it seems to be associated with the nucleolus in the interphase nucleus (Bobrow et al., 1971; Ibraimov, 2017). Whilst the purpose of the chromosomal Qheterochromatin is still in the realms of speculation its unique character, both as an indicator of human and hominoid chromatin and direction of evolution, cannot be doubted (Ibraimov, 2010, 2015).

Data on the distribution of the amount of chromosomal Q-HRs in human populations residing in different climaticand-geographic conditions of Eurasia and Africa, in the newcomers, who have successfully adapted to the harsh environmental conditions of the far North, the high altitude of Pamir and Tien-Shan, as well as in individuals from different age groups and patients suffering from purely "human" forms of pathology (obesity, alcoholism and drug addiction) give rise to doubt in the presumption of genetic inertness of chromosomal Q-HRs (Ibraimov, 2010, 2015, 2016a,b, 2017).

If consider these data as evidence that chromosomal Q-HRs has some selective value, and their amount in the human genome is related to its ability to adapt, for example, to cold and hypoxia, then it probably is easier to perceive our idea of the reason for the merger of the two pairs of acrocentrics in the karyotype of the common ancestors 
We believe that only with the appearance in the population of individuals with different, and especially with a low number of chromosomal Q-HRs the modern man was able to develop and populate the entire land. For this it did not require some unusual revolutionary changes in the genome, it was enough routine chromosomal rearrangements, which have always had a place in the evolution of higher eukaryotes. Therefore, the existence in the genome of a modern human of 46 chromosomes instead of 48 like in the other two apes, the fact is notable, but trivial enough. Here it was important not decrease in the number of chromosomes, but decrease in the number of Q-polymorphic autosomes, which prevented giving birth in the population to the individuals with different amounts of Q-HRs. This became possible after two pairs of acrocentrics bearing Q-HRs with a particularly high frequency in the population disappeared in the genome of the common ancestors.

Perhaps, the events developed in this sequence. In the genome of the common ancestors with 48 chromosomes, the number of Q-HRs was so much that probability of giving birth to the individuals in the population with different and especially with the low number of Q-heterochromatin was extremely low. Thus, for example, the number of chromosomal Q-HRs in the genome of the modern indigenous inhabitants of Africa varies from 1 to $10(m=4.6)$, and natives of the far North of Siberia or the highlanders of Pamir and Tien-Shan are even less, from 0 to 5 ( $m=$ 2.2) (Ibraimov, 1993, 2010, 2011). In chimpanzee the frequency of chromosomal Q-HRs is considerably higher than in man; in man their incidence averages from about 2.9-4.2 (Buckton et al., 1976), while in the chimpanzee it has been estimated to be equal to 8.77 , and to 8.85 . The frequency of Q-HRs 14.8 in gorilla, which is approximately five times the number observed in man. Q-heterochromatin is found at the Y chromosome longarm distal tip in this species. Q-HRs polymorphisms are also evident at the telomeric region of many chromosome arms (see above).

Judging by the distribution of chromosomal Q-HRs in the genome of chimpanzees and gorillas, probability of giving birth to individuals with different and especially with the low number of Q-heterochromatin is virtually eliminated. Perhaps, in the population of the common ancestors the distribution of the number of chromosomal QHRs has occurred close to these two apes, which is not to favour to the birth of individuals with different numbers of Q-heterochromatin in the genome.

As the common ancestors expanded the territory of their habitat at the expense of more severe climatic zones, probability to survive for individuals with the amount of chromosomal Q-HRs close to the modern native Africans may be increased. But at this the number of chromosomes in the genome of born with low amount of Q-HRs still made 48. However, in the population the individuals even with 46 chromosomes were born, including those in which the number of chromosomes was reduced by merging those of those two pairs of acrocentrics, which differed with a particularly high content of Q-HRs. If so, then such individuals could be better adapted to new climatic conditions, to prevail in number in the population, etc., with all the ensuing consequences. In other words, we let in the possibility of coexistence in the human evolution initial stages the individuals with 48 and 46 chromosomes within the same population. Only with the lapse of time, when the number of individuals in the population with 46 chromosomes (where two pairs of high Q-polymorphic acrocentrics formed chromosome 2 of the modern human) the likelihood of their mating began significantly increase. Gradually, these individuals could form a new population on the new territory. But it was the beginning of a new species, appearance, which now calls itself proudly - Homo sapiens sapiens.

\section{References}

Al-Nassar, K. E., Palmer, C. G., Connealy, P. M., \& Pao-Lo, Y. (1981). The genetic structure of the Kuwaiti population. II. The distribution of Q-band chromosomal heteromorphisms. Hum Gene., 57, 423-427.

Andrews, P., \& van Couvering, A. H. (1975). In F. S. Szalay (Ed.), Approaches to Primate Paleobiology (pp. 62105). Karger, Basel.

Arrighi, F. E., \& Hsu, T. C. (1971). Localization of heterochromatin in human chromosomes. Cytogenetics, 10, 81-86.

Bhasin, M. K. (2005). Human population cytogenetics. A review. Int. J. Hum. Genet, 5(2), 83-152.

Bobrow, M., Pearson, P. L., \& Gollacott, H. E. A. C. (1971). Para-nucleolar position of the human Y chromosome in interphase nuclei. Nature, 232, 556-557.

Buckton, K. E., O'riordan, M. L., Jacobs, P. A., Robinson, J. A., Hill, R., \& Evans, H. J. (1976). C-and Q-band polymorphisms in the chromosomes of three human populations. Annals of human genetics, 40(1), 99-112.

Caspersson, T., Zech, L., \& Johansson, C. (1970). Differential binding of alkilating fluorochromes in human chromosomes. Exp Cell Res, 60, 315-319. 
Chiarelli, B. (1962). Comparative morphometric analysis of thr Primate chromosomes. I. Cariologia, 15, 99-121.

Chiarelli, B., \& Lin, C. C. (1972). Comparison of fluorescence patterns in human and chimpanzee chromosomes. Genet Phaenen, 15, 103-106.

Erdtmann, B. (1982). Aspects of evaluation, significance, and evolution of human C-band heteromorphism. Hum Genet, 61, 281-294.

Hamerton, J. L., Klinger, H. P., Mutton, E., \& Lang, E. M. (1963). The somatic chromosomes of the Hominoidea. Cytogenetics, 2, 240-263.

Hsu, T. C., \& Arrighi, F. E. (1971). Distribution of constitutive heterochromatin in mammalian chromosomes. Chromosoma, 34, 243-253.

Ibraimov A. I., \& Mirrakhimov, M. M. (1982d). Human chromosomal polymorphism. II. Chromosomal Cpolymorphism in Mongoloid populations of Central Asia. Hum. Genet., 60, 8-9.

Ibraimov, A. I. (2010). Chromosomal Q-heterochromatin regions in populations and human adaptation. In: MK Bhasin, C Susanne (Eds.): Anthropology Today: Trends and Scope of Human Biology. Delhi: Kamla- Raj Enterprises, pp. 225-250.

Ibraimov, A. I. (2011). Origin of modern humans: a cytogenetic model. Hum Evol, 26(1-2), 33-47.

Ibraimov, A. I. (2015). Heterochromatin: The visible with many invisible effects. Global J Med Res, (C), 15(3), 7-32.

Ibraimov, A. I. (2016a). Chromosomal Q-Heterochromatin Polymorphism in Patients with Alimentary Obesity. Biol Med (Aligarh), 8, 275.

Ibraimov, A. I. (2016b). Chromosomal Q-heterochromatin Regions in Alcoholics and Drug Addicts. Biol Med (Aligarh), 8, 346.

Ibraimov, A. I. (2017). Cell Thermoregulation: Problems, Advances and Perspectives. J Mol Biol Res, 7(1), 42-63.

Ibraimov, A. I., Akanov, A. A., Baygazieva, G. D., \& Meimanaliev, T. S. (2014). Chromosomal Qheterochromatin and age in human population. Journal of Molecular Biology Research, 4(1), 1-9.

Ibraimov, A. I., Akanov, A. A., Meymanaliev, T. S., Karakushikova, A. S., Kudrina, N. O., Sharipov, K. O., \& Smailova, R. D. (2013). Chromosomal Q-Heterochromatin Polymorphisms in 3 Ethnic Groups (Kazakhs, Russians and Uyghurs) of Kazakhstan. International Journal of Genetics, 5(1), 121.

Ibraimov, A. I., Aksenrod, E. I., Kurmanova, G. U., \& Turapov, O. A. (1990). Chromosomal Q-heterochromatin regions in the indigenous population of the northern part of West Siberia and in new migrants. Cytobios, 67(269), 95-100.

Ibraimov, A. I., Kurmanova, G. U., Ginsburg, E., Aksenovich, T. I., \& Aksenrod, E. I. (1989). Chromosomal Qheterochromatin regions in native highlanders of Pamir and Tien-Shan and in newcomers. Cytobios, 63(253), 71-82.

Ibraimov, A. I., Mirrakhimov, M. M., Axenrod, E. I., \& Kurmanova, G. U. (1986). Human chromosomal polymorphism. Human genetics, 73(2), 151-156.

Ibraimov, A. I., Mirrakhimov, M. M., Nazarenko, S. A., Axenrod, E. I., \& Akbanova, G. A. (1982). Human chromosomal polymorphism. I. Chromosomal Q polymorphism in Mongoloid populations of Central Asia. Human genetics, 60(1), 1-7.

Ibraimov, A. I. (1993). The origin of modern humans: a cytogenetic model. Hum Evol, 8(2), 81-91.

Ibraimov, A. I., \& Mirrakhimov, M. M. (1982a). Human chromosomal polymorphism. III. Chromosomal Qpolymorphism in Mongoloids of Northern Asia. Hum Genet, 62, 252-257.

Ibraimov, A. I., \& Mirrakhimov, M. M. (1982b). Human chromosomal polymorphism. IV. Q-polymorphism in Russians living in Kirghizia. Hum Genet, 62, 258-260.

Ibraimov, A. I., \& Mirrakhimov, M. M. (1982c). Human chromosomal polymorphism. V. Chromosomal Qpolymorphism in African populations. Hum Genet, 62, 261-265.

Ibraimov, A. I., \& Mirrakhimov, M. M. (1985). Q-band polymorphism in the autosomes and the Y chromosome in human populations. In A. A. Sandberg (Ed.), Progress and Topics in Cytogenetics. The Y chromosome. Part A. Basic characteristics of Y chromosome (pp. 213-287). Alan R. Liss, Inc., New York. USA. 
John, B. (1988). The biology of heterochromatin. In R. S. Verma (Ed.), Heterochromatin: Molecular and Structural Aspects (pp. 1-147). Cambridge University Press, Cambridge, New York, New Rochelle, Melburn, Sydney.

John, B., \& Micloš, G. L. G. (1979). Functional aspects of satellite DNA and heterochromatin. Int Rev Cytol, 58, $1-114$.

Kalz, L., Kalz-Fuller, B., Hegde, S., \& Schwanitz, G. (2005). Polymorphisms of Q-band heterochromatin: qualitative and quantitative analyses of features in 3 ethnic groups (Europeans, Indians, and Turks). International Journal of Human Genetics, 5(2), 153-163.

Lin, C. C., Chiarelli, B., Boer, L. E. M. de, \& Cohen, M. M. (1973). A comparison of fluorescent karyotype of the chimpanzee (Pan troglodytes) and man. J Hum Ecol, 2, 311-321.

Lubs, H. A., Kimberling, W. J., Hecht, F., Patil, S. R., Brown, J., Gerald, P., \& Summitt, R. L. (1977). Racial differences in the frequency of $\mathrm{Q}$ and $\mathrm{C}$ chromosomal heteromorphisms. Nature, 268(5621), 631-633.

Micloš, G. L. G., \& John, B. (1979). Heterochromatin and satellite DNA in man: properties and prospects. Am J Hum Genet, 31, 264-280.

Miller, D. A., Firschein, 1. L., Dev, V, G., Tantravahi, R., \& Miller, O. J. (1974). The gorilla karyotype: chromosome length and polymorphisms. Cytogenet Cell Genet, 13, 536-550.

Pearson, P. (1973). The uniqueness of the human karyotype. Nobel Symposium 23. Chromosome identification. p. 145-151.

Pearson, P. L., Bobrow, M., Vosa, C. G., \& Barlow, P. (1971). Quinacrine fluorescent in mammalian chromosoms. Nature, 231, 326.

Pearson, P. L., Geraedts, J. P. H., \& van der Liuden, A. G. L. M. (1972). Human chromosome polymorphism: Constitutive heterochromatin in man. Medica Hoechst, 6, 201-215.

Prokofyeva-Belgovskaya, A. A. (1986). Heterochromatic Regions of Chromosomes (in Russian). Moscow, Nauka.

Seuanez, H. (1977). Chromosoms and spermatozoa of the great apes and man. Thesis, Univ. Edinburgh.

Seuanez, H. (1979). The phylogeny of human chromosomes. Springer-Verlag Berlin Heidelberg New York.

Stanyon, R., et al., (1988). Population cytogenetics of Albanians in the province of Cosenza (Italy): frequency of $\mathrm{Q}$ and $\mathrm{C}$ band variants. Int J Anthropol, 3(1), 14-29.

Turleau, C., de Grouchy, J., \& Klein, M. (1972). Phylogenic chromosomique de l'homme et des primates hominiens (Pan troglodytes, Gorilla gorilla and Pongo pygmaeus) essai de reconstitution du caryotype de l'ancetre commun. An. Genet, 15, 225-240.

Verma, R. S. (1988). Heterochromatin. Molecular and Structural Aspects. Cambridge Univ. Press. Cambridge, New York, Sydney.

Verma, R. S., \& Dosik, H. (1980). Human chromosomal heteromorphisms in American blacks. I. Structural variability of chromosome 3 . J Hered, 71, 441.

Weisblum, B., \& de Haseth, P. L. (1972). Quinacrine, a chromosome stain specific for deoxyadenylatedeoxythymidilate-rich regions in DNA. Proc Nat Acad Sci, USA, 69, 629-632.

\section{Copyrights}

Copyright for this article is retained by the author(s), with first publication rights granted to the journal.

This is an open-access article distributed under the terms and conditions of the Creative Commons Attribution license (http://creativecommons.org/licenses/by/4.0/). 